\title{
haben oder sein - avoir oder être Der Einsatz der Auxiliarverben bei der Bildung der zusammengesetzten Vergangenheit im DaF/DaZ- und FLE-Unterricht
}

\section{Bernhard Offenhauser}

The formation of the composite tense forms of the past in German and French is similar regarding the use of the auxiliar verbs. In German as a foreign or second language classes and in French as a foreign language classes (Français langue étrangère), emphasis is laid on the exceptions from the overall rules. However, in beginners' lessons it would be more useful to emphasise the common regularities of auxiliar verb selection valid for both languages. In the following text, it is demonstrated by means of corpus analysis that the shared features of auxiliar selection in both languages indeed prevail on the level relevant for beginners. Exceptions can actually be confined to one single verb, namely to verlassen / sortir which selects haben in German and être in French. The consequences of this state of affairs for foreign language teaching are implemented in form of a "sein = être house" inspired by the so called "être house" which is widely used in FLE teaching.

auxiliary verb - composite perfect - passé compose - corpus analysis - être house - German as a foreign language - French as a foreign language - DeReKo - InterCorp

Die Bildung der zusammengesetzten Zeitformen der Vergangenheit im Deutschen und Französischem verläuft nach ähnlichen Strukturen bezüglich des Einsatzes der Auxiliarverben. Im DaF/DaZ Unterricht und im FLE Unterricht (Français langue étrangère, Französisch als Fremdsprache) konzentriert man sich dabei in hohem Maße auf die Ausnahmen bzw. die Sonderformen. Dagegen wäre es im AnfängerInnenunterricht gerade nützlich, den Transfer von Regularitäten zwischen den beiden Sprachen zu fördern. Im Folgenden wird anhand von Korpusanalysen empirisch belegt, dass im themenrelevanten Kontext tatsächlich die Gemeinsamkeiten in der Auxiliarwahl überwiegen. Die Sonderformen lassen sich im Wesentlichen auf ein einziges Verb einschränken, nämlich auf verlassen / sortir, das im Deutschen das Perfekt mit haben, im Französischen mit être bildet. Die entsprechenden Konsequenzen für den Fremdsprachenunterricht werden in Form eines „sein = être-Haus“ (in Anlehnung an das être-Haus aus dem FLE Unterricht) gezogen, in dem die Verben, die im Deutschen und im Französischen die jeweilige zusammengesetzte Vergangenheit mit sein bzw. mit être bilden, in einer Graphik dargestellt werden.

Auxiliarverb - zusammengesetzte Vergangenheit - Perfekt - passé composé - être-Haus Korpusanalyse - être-Haus - Deutsch als Fremdsprache - Français langue étrangère DeReKo - InterCorp

\section{Einleitung}

Im DaF/Z-Unterricht setzen sich französischsprachige, genauso umgekehrt, im FLE-Unterricht (Français langue étrangère, Französisch als Fremdsprache) deutschsprachige Lernende mit der Perfektbildung bzw. der Bildung des passé 
composé auseinander. Nach eigenen Beobachtungen funktioniert dabei der korrekte Einsatz des Auxiliarverbs, solange die Sonderfälle im Unterricht nicht thematisiert werden. Mit anderen Worten kommt es kaum zu Komplikationen, solange Sonderfälle dem Transfer der Lernenden überlassen werden, respektive die Regel der deutschen Auxiliarverbanwendung im Französischen angewandt wird und umgekehrt. Die Schlussfolgerung aus dieser Beobachtung gilt es in der hier vorliegenden Arbeit anhand von Korpora zu überprüfen, zu bestätigen und daraus didaktische Konsequenzen zu ziehen.

\subsection{Problemstellung und Zielsetzung}

In der vorliegenden Arbeit werden die Gemeinsamkeiten in der Perfektbildung und in der Bildung des passé composés bezüglich des Einsatzes der Auxiliarverben haben und sein bzw. deren französischem Pendants avoir und être aufgezeigt. Der Hypothese nach werden hierbei im Deutschen und im Französischen weitgehend dieselben Unterscheidungen getroffen. Demnach gilt es, einerseits die Lernenden als auch die Lehrenden von der verwirrenden Last der Ausnahmen bzw. der Sonderfälle der Perfektbildung in der jeweiligen Fremdsprache im AnfängerInnenunterricht zu entlasten und mehr den direkten Regeltransfer zwischen dem Deutschen und dem Französischen und umgekehrt zuzulassen. Andererseits ist Ziel dieses Artikels, neue, wenn auch teilweise höchst spezifische Sonderfälle zu eruieren und diese nach der Relevanz im GERS, nach der Frequenz in Sprachkorpora und nach deren Verwendung in beiden Sprachen zu kategorisieren um daraus konkrete didaktische Konsequenzen zu ziehen.

\subsection{Arbeitsgrundlage}

Im vorliegenden Artikel werden, aufbauend auf Lehrwerken, Lernendengrammatiken und Wörterbüchern, gängige Regelwerke im themenrelevanten Kontext zusammengefasst. Die daraus extrahierten Verben werden mit Hilfe von Profile Deutsch den Niveaustufen des GERS zugeordnet. Anhand des deutschen Referenzkorpus DeReKo wird die jeweilige Frequenz ermittelt, um die tatsächliche Bedeutung in der deutschen Sprache festzustellen. Im nächsten Abschnitt gilt es, die Regularitäten und die Sonderfälle bezüglich der Perfekt- bzw. der Passé-ComposéBildung mittels des Parallelkorpus InterCorp anhand einer Verbliste zu belegen, wodurch die Regeln und Ausnahmen in überarbeiteter Form dargestellt werden, nicht zuletzt um die Lernenden, aber auch die Lehrenden von den Sonderfällen zu entlasten. Aus den gewonnenen Erkenntnissen werden als Ziel dieser Arbeit die daraus resultierenden didaktischen Konsequenzen präsentiert. 


\section{Der Vermittlungsweg der Perfektbildung im Unterricht}

Das Perfekt wird in beiden Fremdsprachen relativ früh gelernt, d. h. bereits auf der Niveaustufe A1 ist diese Zeitform mit den bedeutendsten Sonderfällen vollständig eingeführt. Dieses Tempus wird zumeist schrittweise erarbeitet. Üblicherweise werden zuerst die Bildung des Partizip II und die Perfektbildung mit haben bzw. mit avoir thematisiert und geübt, kurz daran anschließend die Sonderfälle mit sein bzw. mit être (vgl. hierfür Runge und Rousseau 2012: 107, 125; Krenn und Puchta 2015a: 111). Im Folgenden wird zunächst auf die jeweiligen ausgangs- und zielsprachlichen Feinheiten bezüglich des Auxiliarverbeinsatzes eingegangen und darauf folgend auf die daraus resultierenden Probleme.

\subsection{Die Bildung des Perfekts im DaF/DaZ-Unterricht}

Die Dudenredaktion (Duden online) gibt als Regel bezüglich der Unterscheidung von haben und sein im themenrelevanten Kontext folgendes an: Nur „intransitive Verben, die den Übergang in einen neuen Zustand bezeichnen, [bilden] das Perfekt mit sein“ (ebd.). An selbiger Stelle wird angeführt, dass ,viele Bewegungsverben [...] das Perfekt sowohl mit haben als auch mit sein bilden“ (ebd.), wobei wenn „diese Verben allerdings mit einer Richtungs- oder Ortsangabe verbunden [werden], [...] nur die Perfektbildung mit sein" möglich ist (ebd.).

Im österreichischen Schulwörterbuch von Duden wird diesbezüglich im Sinne der standardsprachlichen Varietäten im Deutschen präzisiert, wobei angeführt wird, dass „Verben, die eine Körperhaltung ausdrücken, [...] das Perfekt mit sein“ bilden (Duden 2008: 15).

In vielen Lehrwerken wird der Komplexität des Themas insofern begegnet, als Verblisten von Sonderfällen erstellt werden, in denen die Verben, die mit sein im Perfekt gebildet werden, angeführt sind (vgl. hierfür z. B. Götz und Schwarz 2014: 78; Krenn und Puchta 2015b: 130; Runge und Rousseau 2012: 163).

\subsection{Die Bildung des Passé Composé im FLE-Unterricht}

Im FLE-Unterricht (Français Langue Étrangère) wird das passé composé ähnlich wie im DaF/DaZ-Unterricht vermittelt. Zuerst werden die Regelfälle betrachtet, also die Formen, die mit avoir gebildet werden, daran anschließend die Sonderfälle, die mit être gebildet werden (vgl. hierfür Runge und Rousseau 2012: 107, 125; Krenn und Puchta 2015a: 111).

Diese betrifft nach Grevisse und Goosse (1981: 231 f) die pronominalen Verben, die sogenannten verbes réfléchis und einige intransitive Verben, die zumeist eine Bewegung oder eine Zustandsveränderung ausdrücken. Angemerkt wird dazu an selbiger Stelle, dass diese intransitiven Verben mit avoir gebildet werden, wenn sie transitiv benutzt werden (s. dazu auch in Klein und Kleineidam 1988: 71). 
In der Lernendengrammatik von Grevisse und Goosse (1981: 232) werden, um die oben genannte Regel zu konkretisieren, 14 Verben angeführt, die intransitiv benutzt das passé composé mit être bilden. Eine ähnliche Liste, nur um einige Verben erweitert, befindet sich in Callet (2013: 186).

In gängigen Unterrichtswerken am österreichischen Markt wird dem Problem avoir oder être mit Verblisten begegnet, die oftmals graphisch in Form des sogenannten être-Hauses dargestellt werden (Runge und Rousseau 2012: 131). Dabei handelt es sich um eine graphische Darstellung, in der die französischen Verben, die das passé composé mit être bilden, in der Skizze eines Hauses dargestellt sind. Der Fragestellung dieses Artikels nach kann dieses Haus auf den DaF/DaZ Unterricht übertragen werden.

\subsection{Gegenüberstellung der Verben und die Konsequenz: die Nichtausnahmen?}

In diesem Abschnitt wird eine Liste an Verben erstellt, die entweder im Deutschen das Perfekt mit sein bilden oder im Französischen mit être - oder gemäß der Fragestellung dieses Beitrags - an Verben, die in beiden Sprachen mit sein oder être das Perfekt bilden. Als Grundlage für diese Liste werden in Bezug auf das Französische die bereits erwähnte Aufzählung aus Callet (2013: 186) und bezüglich des Deutschen das Österreichische Schulwörterbuch vom Dudenverlag genommen. Die Verben werden nach der deutschen Spalte alphabethisch absteigend geordnet und mit Hilfe der Profile Deutsch CD (Glaboniat 2015) werden die einzelnen Verben der jeweiligen Niveaustufe des GERS zugeordnet. Daran anschließend wird im Korpus DeReKo (Deutsches Referenzkorpus) die jeweilige relative Häufigkeit ermittelt, um feststellen zu können, inwiefern die jeweiligen Verben für den Spracherwerb von Bedeutung sind. Je häufiger die Verben vorkommen, desto früher sollten diese erlernt werden bzw. desto eher sind diese vom Wirrwarr diverser Ausnahmeregelungen zu entlasten.

In Tab. 1 werden die Verben in einer Übersicht dargestellt. In der linken Spalte befinden sich die deutschen Verben, die nach dem Duden (2008: 15) das Perfekt mit sein bilden. Wichtig ist an dieser Stelle zu präzisieren, dass die deutschen Verben in der linken Spalte, die kursiv markiert sind, je nach Verwendungskontext bzw. Varietät, das Perfekt mit sein und mit haben bilden. Von dieser Unterscheidung wird hier abgesehen, da sie im AnfängerInnenunterricht noch von geringer Bedeutung ist.

In der daran anschließenden Spalte wird die jeweilige Sprachniveaustufe für die deutschen Verben dargestellt. In der mittleren Spalte befinden sich nach Callet (2013: 186) die Verben, die im Französischen das passé composé mit être bilden (grau unterlegt) und die Verben, die, im Gegensatz zum Deutschen, die zusammengesetzte Vergangenheit nicht mit être bilden. 
Rechts daran anschließend befindet sich die im DeReKo ermittelte relative Häufigkeit der deutschen Verben, wobei die Abkürzung $p M W$ der Einheit pro Millionen Wörter entspricht. Hierbei ist anzuführen, dass die Zahl 1445,0 pMW die vom Korpus vorgegebene Höchstgrenze darstellt. Der tatsächliche Wert kann, wenn notwendig, anhand der absoluten Zahlen ermittelt werden, die sich in der rechten Spalte befinden.

Eingabe der Abfrage für die Frequenz im folgenden Archiv: W-öffentlich - alle öffentlichen Korpora des Archivs W (mit Neuakquisitionen):

Einstellungen: Optionen > Suche: Zusammenfassung ab 300 nicht angehakt

Begrenzung der Ergebnismenge $>$ alle Treffer

Suche mit Flexionsform: \&verb; z. B. \&ankommen - damit werden alle lemmatisierten Formen gesucht.

\begin{tabular}{|l|l|l|l|l|}
\hline Deutsch & Niveau (GERS) & Französisch & $\begin{array}{l}\text { rel. Häufigk. } \\
\text { [pMW] }\end{array}$ & Treffer \\
\hline ankommen & A1 & arriver & $43,15 \mathrm{pMW}$ & 298.641 \\
\hline aufstehen & & se lever & $18,84 \mathrm{pMW}$ & 130.411 \\
\hline aufwachen & B1/B2 & se réveiller & $4,359 \mathrm{pMW}$ & 30.164 \\
\hline ausgehen & A2/B1 & partir & $56,51 \mathrm{pMW}$ & 391.071 \\
\hline auswandern & B2 & s'expatrier & $4,047 \mathrm{pMW}$ & 28.008 \\
\hline baumeln & - & pendiller & $2,876 \mathrm{pMW}$ & 19.907 \\
\hline begegnen & - & rencontrer & $32,36 \mathrm{pMW}$ & 223.940 \\
\hline bleiben & B1 & rester & $751,3 \mathrm{pMW}$ & 5.199 .237 \\
\hline einschlafen & & s'endormir $^{\prime}$ & $5,105 \mathrm{pMW}$ & 35.332 \\
\hline eintreten & B2 & entrer & $27,87 \mathrm{pMW}$ & 192.894 \\
\hline entstehen & A2 & devenir & $236,0 \mathrm{pMW}$ & 1.633 .262 \\
\hline explodieren & - & exploser & $13,02 \mathrm{pMW}$ & 90.123 \\
\hline fahren & A1/A2 & conduire & $341,9 \mathrm{pMW}$ & 2.366 .463 \\
\hline fallen & B1 & tomber & $414,6 \mathrm{pMW}$ & 2.869 .180 \\
\hline fliegen & A2 & voler & $72,74 \mathrm{pMW}$ & 503.410 \\
\hline folgen & B1/B2 & suivre & $548,7 \mathrm{pMW}$ & 3.797 .634 \\
\hline geboren_sein & A1 & naitre & $104,5 \mathrm{pMW}$ & 722.910 \\
\hline gehen & A1 & aller & $1421,8 \mathrm{pMW}$ & 9.839 .513 \\
\hline gelingen & B1 & parvenir & $115,3 \mathrm{pMW}$ & 798.112 \\
\hline hängen & & pendre & $100,0 \mathrm{pMW}$ & 691.725 \\
\hline hinabsteigen & - & descendre & $0,4956 \mathrm{pMW}$ & 3.430 \\
\hline hocken & B2 & s'accroupir & $5,584 \mathrm{pMW}$ & 38.642 \\
\hline kauern & - & se tapir & $1,463 \mathrm{pMW}$ & 10.125 \\
\hline klettern & s'agenouiller & $5,203 \mathrm{pMW}$ & 36.008 \\
\hline knien & & & 167.537 \\
\hline & & & & \\
\hline
\end{tabular}




\begin{tabular}{|c|c|c|c|c|}
\hline Deutsch & Niveau (GERS) & Französisch & $\begin{array}{l}\text { rel. Häufigk. } \\
\text { [pMW] }\end{array}$ & Treffer \\
\hline kommen & A1 & venir & $1445,0 \mathrm{pMW}$ & 12.051 .424 \\
\hline landen & $\mathrm{A} 2$ & atterir & 93,9 pMW & 649.889 \\
\hline laufen & $A 2$ & courir & $316,9 \mathrm{pMW}$ & 2.192 .869 \\
\hline liegen & $\mathrm{A} 2$ & être allongé & $831,7 \mathrm{pMW}$ & 5.755 .525 \\
\hline lungern & & traînailler & $0,4690 \mathrm{pMW}$ & 3.246 \\
\hline reisen & A1 & voyager & $151,8 \mathrm{pMW}$ & 1.050 .871 \\
\hline rennen & B1/B2 & courir & $89,8 \mathrm{pMW}$ & 621.324 \\
\hline schwenken & & agiter & 7,675 pMW & 53.118 \\
\hline schwimmen & $\mathrm{A} 2$ & nager & $33,06 \mathrm{pMW}$ & 228.798 \\
\hline sein & $\mathrm{A} 1 / \mathrm{A} 2$ & être & $1445,0 \mathrm{pMW}$ & 153.107.136 \\
\hline sich ereignen & - & intervenir & 20,40 pMW & 141.210 \\
\hline sich ereignen & - & advenir & - & - \\
\hline sitzen & $\mathrm{A} 1$ & être assis & $197,5 \mathrm{pMW}$ & 1.366 .836 \\
\hline springen & $\mathrm{A} 1 / \mathrm{A} 2$ & sauter & $45,46 \mathrm{pMW}$ & 314.595 \\
\hline starten & $A 2$ & démarrer & $162,8 \mathrm{pMW}$ & 1.126 .603 \\
\hline stecken & & être coincé & $88,85 \mathrm{pMW}$ & 614.876 \\
\hline stehen & B1 & être débout & $1321,8 \mathrm{pMW}$ & 9.147 .547 \\
\hline steigen & B1 & monter & $264,6 \mathrm{pMW}$ & 1.831 .419 \\
\hline sterben & $\mathrm{A} 2$ & mourir & $150,0 \mathrm{pMW}$ & 1.038 .070 \\
\hline treten & B1 & botter & 224,4 pMW & 1.553 .006 \\
\hline $\begin{array}{l}\text { unvermutet } \\
\text { auftreten }\end{array}$ & - & survenir & - & - \\
\hline verbrennen & B1/B2 & brûler & $39,74 \mathrm{pMW}$ & 275.015 \\
\hline verlassen & B1/B2 & sortir & $131,6 \mathrm{pMW}$ & 910.924 \\
\hline versterben & - & décéder & $10,02 \mathrm{pMW}$ & 69.320 \\
\hline vorbeigehen & - & passer & 4,337 pMW & 30.018 \\
\hline wachsen & A2/B1 & croître & $146,6 \mathrm{pMW}$ & 1.014 .734 \\
\hline wandern & B1 & randonner & $35,07 \mathrm{pMW}$ & 242.713 \\
\hline zurückkehren & - & rentrer & $41,17 \mathrm{pMW}$ & 284.914 \\
\hline zurückkehren & - & retourner & - & - \\
\hline zurückkommen & - & revenir & 9,91 pMW & 68.601 \\
\hline
\end{tabular}

Tab. 1: Verben, die mit sein bzw. mit être das Perfekt bilden und die absolute und relative Häufigkeit im Korpus DeReKo. Die französischen Verben, die grau unterlegt sind, bilden das passé composé mit être. ${ }^{1}$

1 Arbeitsgrundlage: eigene Darstellung; Deutsche Verben: Duden (2008: 15), Krenn und Puchta (2015b: 130); französische Verben: Callet (2013: 186); Sprachniveauzuordnung: Glaboniat (2015); Frequenz: Institut für deutsche Sprache (2016). 


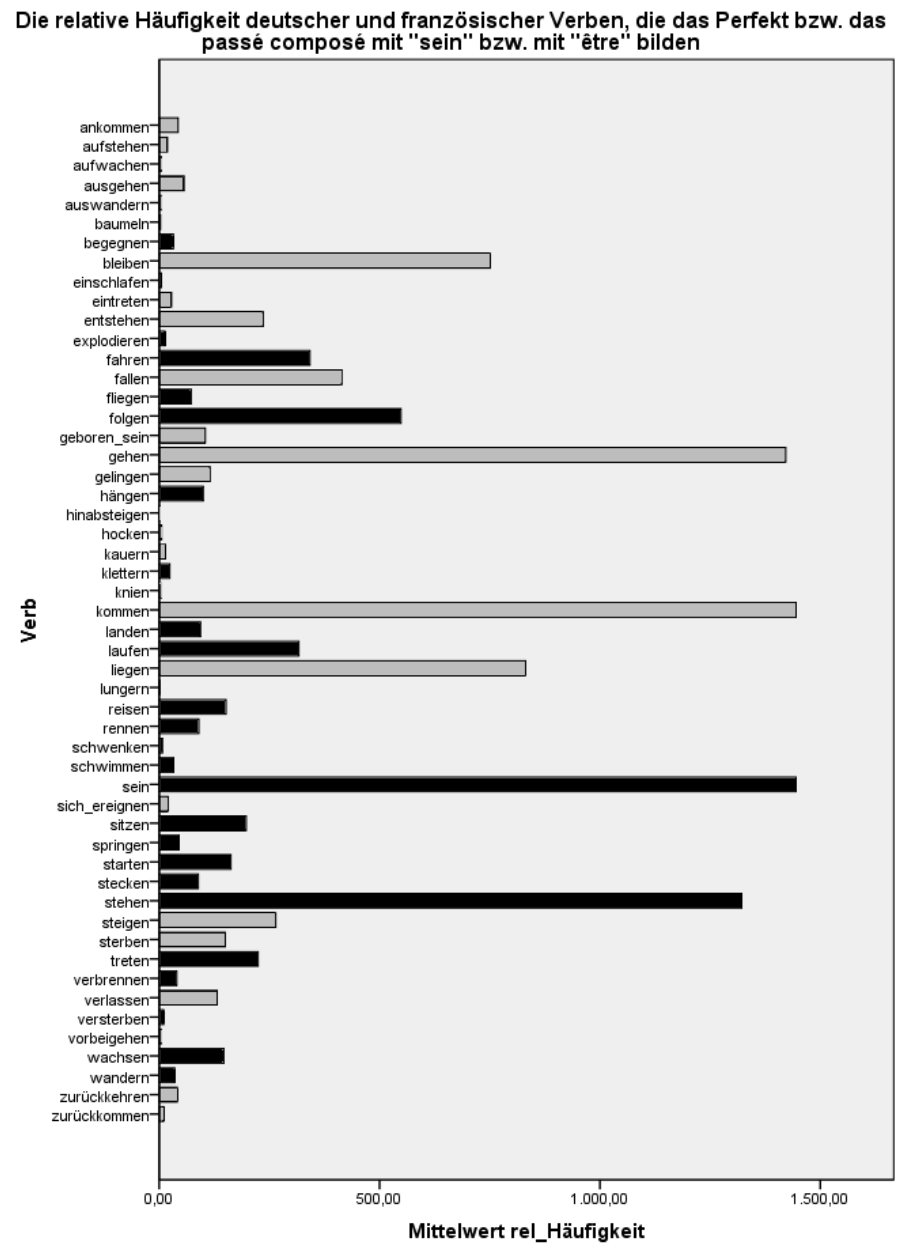

Abb. 1: Die relative Häufigkeit ermittelt mit dem Korpus DeReKo deutscher und französischer Verben, die das Perfekt bzw. das passé composé mit sein bzw. mit être bilden. ${ }^{1}$

Um die Frequenz besser verdeutlichen zu können, wurde aus Tabelle 1 die oben angeführte Graphik (Abb. 1) erstellt. Hierbei sind die deutschen Verben aufgelistet. Die horizontalen Balken geben die relative Häufigkeit an. Die schwarzen Balken

1 Eigene Darstellung. Arbeitsgrundlage: Deutsche Verben: Duden (2008: 15); Krenn und Puchta (2015b: 130) französische Verben: Callet (2013: 186), Sprachniveauzuordnung: Glaboniat (2015); Frequenz: Institut für deutsche Sprache (2016: online). 
symbolisieren die Verben, die im Deutschen das Perfekt mit sein bilden, die grauen Balken die Verben, die im Französischen das passé composé mit être bilden.

Aus Abb. 1 ist somit abzulesen, dass über die Frequenz der Verben, wie sie in DeReKo vorzufinden sind, kaum eine eindeutige Antwort geliefert werden kann, ob nun ein Verb abhängig von der statistischen Häufigkeit die zusammengesetzte Vergangenheit mit sein bzw. mit être bildet. Diese Erkenntnis wird durch das folgende Histogramm bekräftigt. Daraus ist, in anderen Worten, lediglich abzulesen, dass Verben aus Tab. 1 mit einer geringen relativen Häufigkeit öfter auftreten (linke Balken) als die hochfrequenten (rechte Balken). Folglich kann man den Schluss ziehen, dass aus der geringen Menge der Verben, die das zusammengesetzte Perfekt mit sein bzw. mit être bilden, die niedrigfrequenten Verben öfter vertreten sind als die hochfrequenten, wodurch folglich keine eindeutige Pauschalregel für den Fremdsprachenunterricht abgeleitet werden kann.

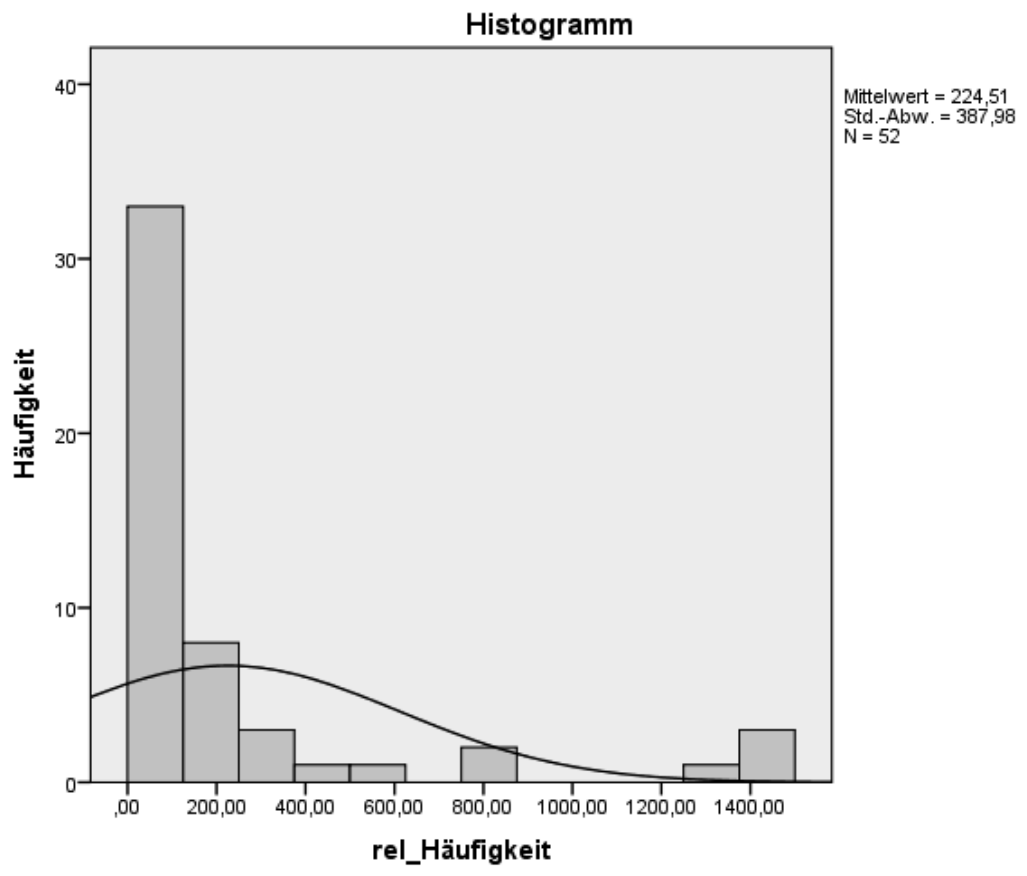

Abb. 2: Histogramm der Verben, die die zusammengesetzte Vergangenheit mit sein bzw. mit être bilden. ${ }^{1}$

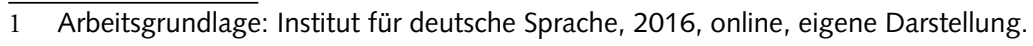


Es kann aber durch Abb. 1 verdeutlicht werden, was bereits in Tab. 1 ersichtlich ist: Beinahe alle untersuchten Verben, die im Französischen das passé composé mit être bilden, bilden im Deutschen das Perfekt mit sein.

Für genauere Untersuchungen in diesem Bereich müsste ebenso ein französisches Korpus befragt werden (z. B. Frantexte), wodurch die Frequenz der Verben, die im Französischen das passé composé mit être bilden, ebenso ermittelt werden kann. Weiters ist das Problem mit der direkten Übersetzung einzelner Wörter zu nennen. Im themenrelevanten Kontext hat dies zu bedeuten, dass, abhängig von der Übersetzung bzw. dem Verwendungskontext und der Varietät, einzelne Wörter mit sein bzw. mit être die zusammengesetzte Vergangenheit bilden können oder eben mit haben bzw. mit avoir.

Trotz all dieser Hürden ist auf alle Fälle das bisherige Ergebnis zu unterstreichen, dass erstens die Frequenz wenig über den Einsatz des Auxiliarverbs aussagt. Zweitens ist die Erkenntnis von Bedeutung, dass beinahe alle untersuchten französischen Verben, die mit être das passé composé bilden auch im Deutschen das Perfekt mit sein bilden.

\section{Belege aus dem Parallelkorpus}

In diesem Abschnitt gilt es, die in Tab. 1 angeführten französischen Verben hinsichtlich ihres Gebrauchs der Auxiliarverben in der zusammengesetzten Vergangenheit zu überprüfen. Als Datengrundlage werden die Erkenntnisse aus dem Parallelkorpus InterCorp genommen, mit Hilfe dessen die französischen Verben, die das passé composé mit être bilden, mit dem deutschen Pendant verglichen werden. Der oben erworbenen Feststellung nach bildet das jeweilige deutsche Äquivalent das Perfekt mit sein, was es hier zu belegen gilt.

Einstellungen für das Parallelkorpus InterCorp:

- Corpus: InterCorp v9 - German

- Query Type: Word Form: die bestimmte, eingegebene Wortform wird gesucht, d. h. die im Deutschen unveränderliche Form des Partizips II, z. B. ,angekommen“"

- Corpus: InterCorp v9 - French

- Query Type: Word Form: ,participe passé*`, z. B. arrive“; der Asterisk muss zum französischen Partizip II zusätzlich eingegeben werden, da es veränderlich ist und ein - $e$ und/oder ein $-s$ angehängt werden kann.

- „Query Type: Word Form“ ist zu wählen, damit gezielt nach dem deutschen Pendant gesucht wird. 
Corpus: InterCorp v9 - German

\section{Search in the corpus}

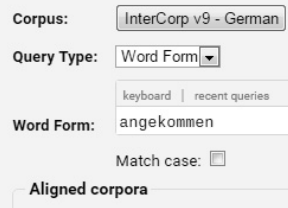

Abb. 3: Einstellungen für die Suchanfrage in kontext mit dem Beispiel ankommen arrivé. $^{1}$

\subsection{Die Ergebnisse:}

Aus der Korpusrecherche wurden jeweils drei repräsentative Ergebnisse ausgewählt, die die Transfermöglichkeit bei der Auxiliarverbenanwendung zeigen. Die Verben wurden aus Tabelle 1 entnommen. Die gesamten Ergebnisse können unter dem folgenden Link angesehen und heruntergeladen werden:

http://bit.do/Ergebnisse_Parallelkorpus_Aux_verben

Es konnten der Hypothese nach die Anwendung und somit auch die Transfermöglichkeit der Auxiliarverben belegt werden. Sechs Abweichungen waren dennoch festzustellen, die im Folgenden näher betrachtet werden.

Für die Verben auswandern - expatrier, hocken - accroupir und knien-agenouiller konnten keine Belege gefunden werden, was mit der in Tabelle 1 angeführten niedrigen Frequenz zu erklären ist. Damit einhergehend sind diese Verben für den AnfängerInnenunterricht von untergeordneter Bedeutung, zumal sie nach Profile Deutsch der Niveaustufe B2 zugeordnet sind. Die Verben kauern - tapir und ereignen

1 Arbeitsgrundlage: Dovalil et al. (2016), eigener Screenshot. 
- intervenir bilden im Deutschen im Gegensatz zum Französischen das Perfekt mit haben. Diese Sonderfälle sind aber wiederum wegen der geringen Frequenz zu vernachlässigen. Für diese Verben kommt hinzu, dass es keine Zuordnung nach den Niveaustufen im GERS gibt.

Somit hat sich durch die Korpusabfrage herausgestellt, dass die einzige themenrelevante Abweichung das Verb verlassen - sortir ist. Im Deutschen bildet verlassen das Perfekt mit haben, im Französischen bildet das Pendant sortir das passé composé mit avoir. Die besondere Bedeutung kommt diesen Verben sowohl durch die hohe Frequenz im DeReKo mit einem Wert von 131,6 pWM zu als auch durch die Zuordnung auf den Niveaustufen B1 und B2.

Als Kritik an dieser Gegenüberstellung ist anzuführen, dass wenige exemplarische Beispiele, wie sie im oben angeführten Link abrufbar sind, bei weitem nicht die gesamte deutsche und französische Sprache repräsentieren. Dem könnte entgegengehalten werden, dass nur die bereits in Lernendengrammatiken verschriftlichten Tatsachen anhand eines Korpus zu belegen bzw. unter dem Blickwinkel des DaF/DaZund FLE-Unterrichts mit Fokus auf den AnfängerInnenunterricht vergleichend darzustellen waren.

Weiters kann angemerkt werden, dass nicht alle Beispiele die reine Form des Perfekts bzw. des passé composé darstellten, sondern andere Zeitformen, wie z. B. das Plusquamperfekt oder das conditionnel du passé, die aber die gleichen Regularitäten der Auxiliarverbunterscheidung aufzeigen.

Dennoch kann zusammenfassend ausgesagt werden, dass die oben angeführte Theorie mit drei Ausnahmen belegt werden konnte und somit zwangsläufig Konsequenzen für den Fremdsprachenunterricht zu ziehen sind.

\subsection{Zusammenfassung der Ergebnisse - Didaktische Konsequenzen}

Das Ergebnis der Analyse aus dem Parallelkorpus ist folgendermaßen darzustellen: Alle Verben, die im Französischen das passé composé mit être bilden, bilden das Perfekt im Deutschen mit sein, bis auf drei Ausnahmen, wobei für den jeweiligen Fremdsprachenunterricht, v. a. auf den Niveaustufen bis B2 nur ein Verb dieser Regularität widerspricht: sortir mit der Bedeutung verlassen. Dieses wird im Französischen mit être gebildet, im Deutschen aber mit haben. Weiters sind zwei niederfrequente Verben zu nennen, die ebenfalls dieser Regularität nicht entsprechen: se tapir - kauern und intervenir - sich ereignen.

Somit lassen sich für deutschsprachige Französischlernende folgende Erleichterungen formulieren: Alle Verben, die im être-Haus (Runge und Rousseau, 2012: 125) vorkommen, werden, analog zum deutschen Perfekt, im passé composé mit être gebildet, außer dem Verb verlassen, welches im Deutschen mit haben das Perfekt bildet, das französische Pendant sortir aber mit être. Die graphische Umsetzung des neuen être-Hauses, das sein = être-Haus sieht folgendermaßen (Abb. 4) aus: 


\section{Das sein $=$ être Haus}

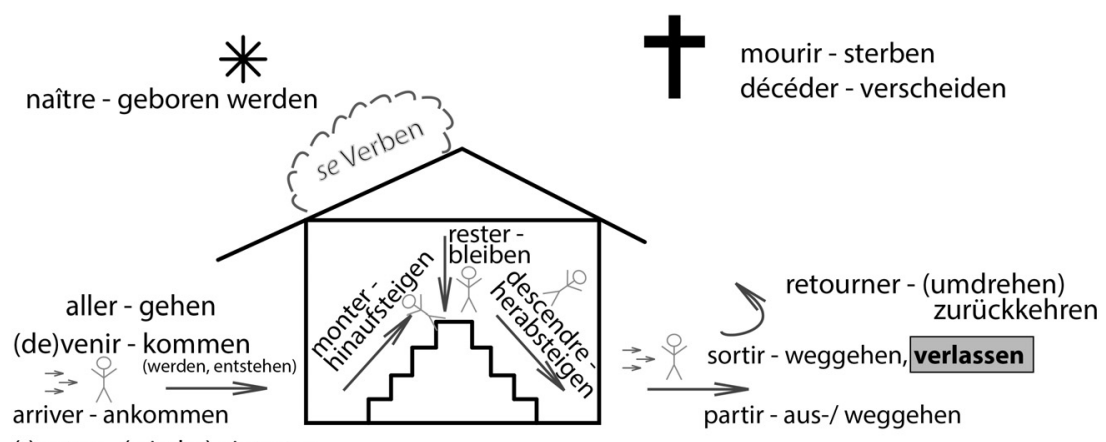

(r)entrer - (wieder) eintreten

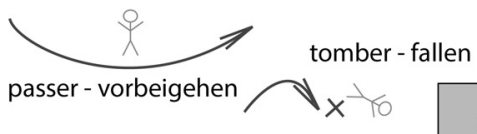

sein $=$ être

außer bei sortir

Abb. 4: Die didaktische Konsequenz aus den Erkenntnissen der Korpusanalyse: das sein = être Haus (eigene Darstellung).

Neben den Verben, die in Abb. 4 dargestellt sind, bilden auch deren französische Komposita das passé composé mit être, wodurch alle drei in der Korpusanalyse herausgefilterten Sonderfälle abgedeckt sind.

Eine Möglichkeit der Didaktisierung ist, einfache Verblisten zu erstellen, die einerseits die Gemeinsamkeiten, andererseits die Unterschiede der Perfekt- bzw. passé composé Bildung gegenüberstellen. Hierbei kann die Frequenz berücksichtigt werden, wobei die niederfrequenten Verben in unteren Lernniveaus weggelassen und nur die hochfrequenten thematisiert werden.

Für gezielte didaktische Umsetzungen, die auf die korrekte Anwendung von avoir und être abzielen, sind bewährte Lückentexte zu empfehlen, wobei es hier von Vorteil ist, die Unterscheidung zwischen den Auxiliarverben nicht zu thematisieren, sondern nur anzugeben, dass die Bildung des passé composé gleich wie jene des deutschen Perfekts funktioniere. In der Anfangsphase wird somit von den Lernenden zwangsläufig entdeckt, dass es keine Sonderformen bzw. Ausnahmen gibt. Hierbei ist zu empfehlen, dass sortir noch weggelassen wird und erst in späteren Übungen als einzige Ausnahme thematisiert wird. 


\section{Zusammenschau und Schlussfolgerung}

In der vorliegenden Arbeit konnte mittels Korpusanalysen belegt werden, dass deutschsprachige FLE-Lernende im Bereich der Bildung des passé composé, respektive in der korrekten Anwendung der Auxiliarverben avoir und être von verwirrenden Ausnahmen bzw. Sonderformen im AnfängerInnenunterricht entlastet werden können, indem ihnen der Regeltransfer vom Französischen ins Deutsche und umgekehrt, vom Deutschen ins Französische zugestanden wird. Zunächst wurde anhand des deutschsprachigen Korpus DeReKo die Frequenz der deutschen Verben, die das Perfekt mit sein bilden, ermittelt, um die jeweilige Notwendigkeit der Verben für gewisse Niveaustufen nach dem GERS zu rechtfertigen bzw. zu belegen (Tab. 1). Weiters wurden die französischen Verben markiert, die das passé composé mit être bilden. Im nächsten Schritt wurden die französischen Verben mit den deutschen Verben im Parallelkorpus InterCorp in Hinblick auf die themenrelevante Anwendung des Auxiliarverbes verglichen. Hierbei konnte die These verifiziert werden, dass alle Verben, die im Französischen das passé composé mit être bilden, auch im Deutschen im Perfekt sein verlangen, wobei drei Abweichungen von dieser Erkenntnis belegt werden konnten.

Daraus kann eindeutig die didaktische Konsequenz gezogen werden, dass den FLE-Lernenden zunächst die Chance eingeräumt werden muss, die Regel der Auxiliarverbanwendung vom Deutschen ins Französische zu transferieren und in einem nächsten Schritt, nachdem ausreichend gezielt die Passé-Composé-Bildung geübt wurde, erst das sein = être-Haus präsentiert wird, in dem die relevanten Verben inklusive eines Sonderfalls angeführt sind.

\section{Literaturverzeichnis}

Callet, Stéphanie (2013): Répertoire des difficultés du français. Vérifier, comprendre, appliquer. Grenoble, Presses Univ. de Grenoble.

Duden (2008): Duden. Das große österreichische Schulwörterbuch. Auf Grundlage der amtlichen Rechtschreibregeln. Mannheim, Wien u. a., Bibliograph. Inst. \& F. A. Brockhaus.

Glaboniat, Manuela (2015): Profile deutsch. Gemeinsamer europäischer Referenzrahmen. Lernzielbestimmungen, Kannbeschreibungen, kommunikative Mittel. Niveau A1-A2, B1B2, C1 - C2. [CD-ROM Version 2.0 mit Begleitbuch]. München, Klett-Langenscheidt.

Götz, Gudrun / Schwarz, Eveline (2014): Von Null auf Deutsch. Deutsch als Zweitsprache für Jugendliche von 11 bis 15, Niveau A1. 4. Aufl. Linz, Veritas-Verl.

Grevisse, Maurice / Goosse, André (1981): Nouvelle grammaire française. 2. Aufl. Stuttgart, Klett.

Káňa, Tomáš (2014): Sprachkorpora in Unterricht und Forschung DaF/DaZ. Brno, Masarykova Univ.

Klein, Hans-Wilhelm / Kleineidam, Hartmut (1988): Französische Grundgrammatik. Für Schule und Weiterbildung. Stuttgart, Klett.

Krenn, Wilfried / Puchta, Herbert (2015a): Ideen 1. Deutsch als Fremdsprache. 2. Aufl. Ismaning, Hueber. 
Krenn, Wilfried / Puchta, Herbert (2015b): Ideen 2. Deutsch als Fremdsprache. 2. Aufl. Ismaning, Hueber.

Runge, Annette / Rousseau Pascale (2012): Perspectives Autriche A1 - A2. Lehr- und Arbeitsbuch. Linz, Veritas-Verl.

\section{Verwendete elektronische Korpora und Internetquellen}

DeReKo: Institut für Deutsche Sprache (2016): Deutsches Referenzkorpus / Archiv der Korpora geschriebener Gegenwartssprache 2016-II. Institut für Deutsche Sprache. Mannheim. Online verfügbar unter www.ids-mannheim.de/DeReKo, Release vom 30.09.2016, zuletzt geprüft am 01.01.2017.

Duden online: Perfektbildung mit „haben“ oder „sein“. http://www.duden.de/sprachwissen/ sprachratgeber/perfektbildung-mit--em-haben--em--oder--em-sein--em- [01.01.2017].

InterCorp: Dovalil, Vít / Káňa, Tomáš / Peloušková, Hana / Zbytovský, Štěpán / Vavřín, Martin (2016): Korpus InterCorp - němčina, verze 9 z 9. 9. 2016. Ústav Českého národního korpusu FF UK. Praha. Online verfügbar unter http://www.korpus.cz.

Bernhard Offenhauser

KF Universität Graz

Institut für Pädagogische Professionalisierung

Strassoldogasse 10/1

8010 Graz

E-Mail: bernhard.offenhauser@uni-graz.at 